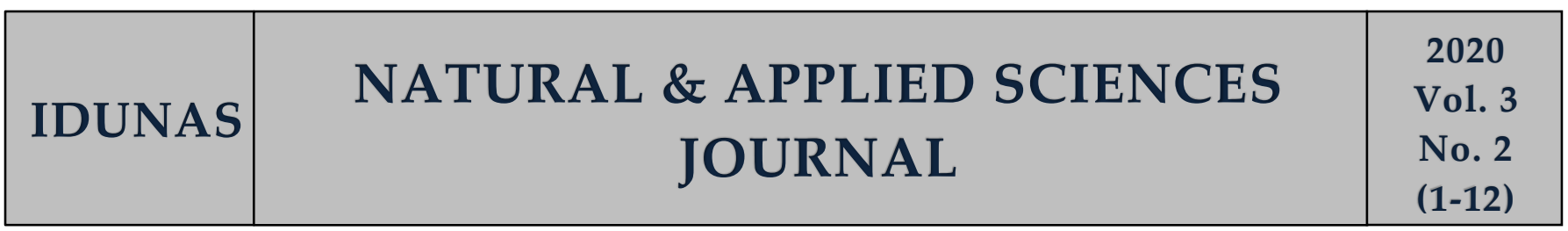

\title{
A Novel Hybrid: Neuro-Immuno-Engineering
}

\author{
Review Article \\ Arzu L. Aral ${ }^{1 *(D)}$, Gönül Ö. Peker² ${ }^{\text {iD }}$ \\ ${ }^{l}$ Department of Immunology, Faculty of Medicine, Izmir Democracy University, Izmir, Turkey \\ ${ }^{2}$ Department of Physiology, Faculty of Medicine, Ege University, Izmir, Turkey (retired)
}

\begin{abstract}
Author E-mails arzuaral@gmail.com

*Correspondance to: Assoc. Prof. Dr. Arzu Latife Aral, Department of Immunology, Faculty of Medicine, Izmir Democracy University, Izmir, Turkey

Tel: +902322601001

DOI: $10.38061 /$ idunas.754647
\end{abstract}

Received:18.06.2020; Accepted:01.12.2020

\section{Abstract}

Although the central nervous system has been known as immune-privileged for many decades, the psychoneuro-endocrine-immune relationships studied in integrity, in recent years has opened a new era called neuroimmunology. Illumination of the bi-directional cross-talk between immune and central nervous systems, both of which are of cardinal importance for homeostasis, survival, progress and wellbeing, and, is highly expected to provide an integrated understanding of neuropathological and degenerative processes. Bioengineering is another novel inter-discipline, which has been developing with great momentum recently. Adaptability, ownership, and mastery of the recipient and the durability and optimal performance of the devices used, seem to be the outmost priority requirement for success. In the context of translational medicine, collaboration between medicine with bioengineering, systems engineering, and material science is definitely the first inevitable requirement for survival and progressive development in the next century. Keywords: Neuroimmunology, neuro-engineering, immuno-engineering, neuro-immune homeostasis

\section{INTRODUCTION}

Restoring and enhancing lost structures and their optimal function but also traveling into the somewhat unknowns of the body with "brain-inspired technics" needs a synergy between living and artificial bio-mimetic systems. Bio-engineering is the discipline resulting from the merge of biology and electronics, and it effectively impacts both individuals' and nations well-being and welfare. A wide variety of bio-materials are used in the central nervous system (CNS) including drug or gene carriers, scaffolds, chips, neural electrodes, and mini labs in site, but also living robots and intelligent neuro-prostheses. These technologies need to be as "near-physiologic" as possible, energy efficient to be handy, and accepted and tolerated by the defence systems of our organism. Additionally, diagnostic and therapeutic tools, and "lab- 
on-chip" methods for research open another vast field to investigate the interesting interactions with or within biology.

Bio-electronics is interested in a range of topics at the interface of biology and electronics. Electronic technics help to analyze biological materials on the cellular and subcellular level. This combination has a wide range of application area in biology, medicine, and even security. Physically interfacing electronic devices with biological systems is also a focus area of bio-electronics (e.g., brainmachine, cell-electrode, or protein-electrode). Assistive technologies for individuals with brain and nervous system disease or injury such as paralysis, blindness and deafness, and new technologies for protein structure-function measurements are also among these areas (Walker et al., 2009).

In addition to biomaterials and bioelectronics, there is another new concept called "bio-hybrid". This system is "formed by at least one natural and one artificial entity that establish close physical interactions at the molecular, cellular, or systems level, eventually leading to information flow and processing in one or both directions" (Vassanelli and Mahmud, 2016).

Whether synthetic or hybrid, such biomechanical devices need to be accepted both by the immune system and by the community, thru overcoming biological, ethical, and social concerns as "foreign". This is only possible with a successful communication within social or biological systems. In this context, it is important to understand the bi-directional cross-talk between the immune system and the CNS to improve the success of neuro-immuno-engineering.

"The overarching technical drivers pushing bioelectronics are the constant advances in semiconductor technology and in surface chemistry related to the interface of biology and man-made devices" (Walker et al., 2009). Both efforts for understanding biological systems and processes at the macro- to nanoscale, and the huge challenge of our century's cross-disciplinary research involving electrical engineering, medicine, biology, chemistry, physics and material science are growing rapidly. In contrast to decades of reductionist biology, today, "systems biology", which "embodies a new perspective from which to view biological systems and knowledge culled from its endeavours leading to advances in medicine and security". This new approach in biomedical research aims to perceive the larger picture by putting all pieces together from the level of organism, tissue, cell, and subcellular organelle to molecule (Walker et al., 2009).

\section{EXCLUSIVE IMMUNITY OF THE NERVOUS SYSTEM}

Sensing and recognition of endangerment, alarming other homeostatic and organ systems for coping with the particular threat, and information storage and retrieval capacity are indispensable for life. Two major unique systems sharing this privilege are the nervous and immune systems. The nervous system and the brain in particular have been considered as "immune privileged" since 1940s. Privilege of immunity is a concept that involves highly controlled maintenance of adaptive immunity and inflammation. However, existence of lymph drainage in the CNS, passage of T and B lymphocytes through blood brain barrier (BBB) and the antigen processing capacity of microglia showed that it actually is not. And today, the bidirectional crosstalk between these systems is a key tool to explain some homeostatic but also pathological conditions (Louveau et al., 2015).

\section{Fundamentals of the Immune System}

The immune system defends our body against foreign invaders such as bacteria, viruses, fungi, parasites, and even allergens. It may recognize the non-self, such as an infectious agent or a damaged cell, by pattern recognition receptors. After assuming it as dangerous, immune system starts to fight this agent recruiting its tools and mechanisms. There are two main components of the immune system called innate and adaptive systems, and they both use humoral and cell mediated immunity to perform their function. Humoral immunity recruits antibodies, cytokines and other soluble mediators, while cellular immunity use many different types of cells as its warriors. 
The first response of the immune system to non-self is established by the innate immunity. Main actors of innate immunity are neutrophils, mast cells, macrophages, natural killer (NK) cells, basophils and eosinophils. When the non-self is not sufficiently inactivated by the innate system, the adaptive immune system starts to fight using its tailor-made warriors called lymphocytes. T helper lymphocytes are divided mainly into two subgroups called pro-inflammatory Th1 and anti-inflammatory Th2. Another group of lymphocytes, named cytotoxic T cells, play role in cellular killing mechanisms, while helper T cells produce pro- or anti-inflammatory cytokines depending on their profile. The main goal of B lymphocyte is to produce antibodies, which are the main tools of humoral immune system. Dendritic cells are professional antigen presenting cells (APC), aiming the T lymphocytes. With the help of the costimulatory molecules expressed as secondary signals by the T cells, an adaptive immune response moves towards the target. One of the most unique characteristics of the immune system is memory. So once faced, it may recognize the danger and when exposed again, it may retrieve the necessary information more rapidly. Immune system should react to the pathogens or cancer cells properly. All these responses should be in control to maintain an immune homeostasis. When this control breaks down, either an excessive immune response to the pathogens or a regular response to self may cause autoimmune disorders. The list and information about main cells of innate and adaptive immunity are given in Table 1.

\section{Glial cells: Friends or Foes?}

The immune activity in the brain has its own rules in addition to the peripheral ones, and these rules are brought to life with both cellular and humoral actors of immunity. In addition to peripheral cells, which monitor the body regularly to detect and destroy infected or transformed cells, a second group called resident immune cells are mainly responsible for the homeostatic processes in CNS (David et al., 2015).

Glial cells are non-neuronal cells in central and peripheral nervous systems (Figure 1). Since they do not produce electrical impulses, for many years glial cells had unfortunately been identified as just "glue cells" owing to their sole and passive task of so-called physical support to neurons. Later however, there had been tremendous amount of contrary data to change this understanding, especially following the histological identification of Einstein's post-mortem brain. According to Diamond's studies performed on the thick slices from a single region of the genius' brain, glial cell counts were much higher compared to those of his regular and healthy age-matched controls (Diamond et al., 1985). In addition to providing mechanical and metabolic support, and protection for neurons, glial cells also maintain messenger homeostasis and endurance of immune activity via soluble mediators such as cytokines and chemokines in the brain (Ramesh et al., 2013). Glial cells perform a vital role in establishing and maintaining the function of the nervous system. Brain's exclusively own and particularly nocturnal glial cleansing machinery called the "glymphatic system" plays a major role in draining the environmental metabolites and possible toxins during sleep, also functioning as the determinant of the fate of neurons via apoptosis or else following injury or neuropathology (Jessen et al., 2015).

There are mainly four different types of glial cells called microglia, astrocytes, oligodendrocytes and ependymal cells (Figure 1). Astrocytes provide protective and metabolic support for neurons with some of the factors called as gliotransmitters while "microglia are involved in synaptic pruning, canvassing extracellular space and phagocytosing apoptotic cells" (Sochocka et al., 2017). Other group of the glial cells called oligodendrocytes also support neurons metabolically and they provide a source of myelin, which surrounds nerve cell axons to insulate them, and increase the rate at which electrical impulses are passed along the axon (Bean et al., 2007). Loss of the trophic support of the glial cells may cause excitotoxicity, weakening of synaptic plasticity, inflammation, and loss of myelination. All these negative effects impair neuronal activity, ultimately affecting behavior (Elsayed and Magistretti, 2015).

Under homeostatic conditions, the brain hosts several heterogeneous populations of myeloid cells (Prinz and Priller, 2014), which may be classified as microglia, meningeal macrophage, choroid plexus macrophage and perivascular space macrophage. Microglia are responsible for both mediating healthy 
function and initiating inflammatory cascades. "While normal inflammation is a tissue healing response; sustained or situationally inappropriate inflammation can exacerbate or directly facilitate the death of adjacent neural and glial cells" (Neher et al., 2012; Brown et al., 2015; Tsui et al., 2019). In the case of injury, microglia stimulate astrocytes to generate a glial scar by differentiating, migrating, proliferating, and secreting fibrous sugar-based matrices. This partitions the injury and poses a physical and chemical barrier preventing axonal regeneration. "The environment within the scar is often dominated by reactive microglia and astrocytes that create cytotoxic conditions, which in turn would signal the death of neurons, first causing the loss of proximal biological circuitry, and later resulting in locomotor and cognitive deficits" (Gaudet and Fonken, 2018; Tsui et al., 2019). Therapeutic devices implanted in the CNS need attenuated microglial activity to improve the lifetime. Biomaterial design and engineering needs immune modulatory approaches to improve device compatibility.

\section{NEUROIMMUNOLOGY}

Neuroimmunology is a broad area playing a major role in achieving a healthy life. Aberrant synaptic pruning during development, obscure modulation of the brain-blood barrier (BBB) for immune cell recruitment, axonal degeneration and demyelination, cellular repair and regeneration, and immune mediated aggregate removal, especially in the late phase of life are several of the conditions where a balanced neuro-inflammation is absolutely necessary (Kierdorf and Prinz, 2017). A daring approach briefly defined as psycho-neuro-endocrino-immunology is interested in these mechanisms and their complicated interactions, which can be divided into several areas of research, and may be depicted as the studies of the following avenues (Kipnis and Filiano, 2017):

a) Bidirectional information trafficking between immune and nervous systems under physiological and pathological conditions and their impact on neural activity

b) The role of microglia in fine-tuning of developing neuronal circuits as well as in the maintenance of neuronal synapses.

c) 'Protective immunity', addressing beneficial immune infiltrates into the CNS following injury and in neurodegenerative diseases"

d) The mechanisms underlying the gut-immune-brain axis (Kipnis and Filiano, 2017).

Neural influences on the immune system are effective via neurotransmitters and neurotrophic growth factors, including NGF and BDNF. There are also hormonal influences acting via epinephrine, norepinephrine, and glucocorticoids (ThyagaRajan and Priyanka, 2012). Oxytocin, a remarkable hormone produced by neurohypophysis, and melatonin, another important hormone produced by epiphysis have very significant regulatory effects on the immune system (Li et al., 2017, Carrillo-Vico et al., 2013). On the other hand; understanding the modulation of nerve cell function by immune and inflammatory mediators such as cytokines, and reactive oxygen and nitrogen species are important mechanisms to fill up the gaps in the neuroimmunologic network. Other physical similarities common to the nervous and immune systems in terms of communication can be stated as synapses and ion channels, both of which are also considered remarkable in understanding the complicated connections, relations, and cross-talks (Hu et al., 2015).

\section{NEUROINFLAMMATION}

In a variety of neurological diseases including cerebrovascular disease (e.g. stroke), neurodegeneration (e.g. Alzheimer's disease), demyelinating diseases (e.g. Multiple Sclerosis) and malign neoplasms, neuroinflammation is an important factor playing role in pathophysiology. Different cell types and molecular mediators of the immune system participating in homeostasis, immune surveillance, regeneration, and repair at controlled situation, may lead to damage of brain tissue under pathological conditions (Figure 2). Activation of microglia, accompanied mostly by BBB breakdown, cytokine and 
chemokine release and blood-borne leukocyte infiltration is one of the hallmarks of neuroinflammation (Pulli and Chen, 2014). Myeloid cells may cause to damage to myelin sheath and neurons through oxidative stress, phagocytosis and protein damage, while lymphocytes may stimulate antibody-dependent cytotoxicity (Pulli and Chen, 2014).

"Neuroinflammation is a highly relevant diagnostic and therapeutic target, but several characteristics of the brain such as BBB and cranial bones, which complicate the access to the brain, make both goals more difficult than at other sites in the body" (Pulli and Chen, 2014). Additionally, the limited regeneration capacity of the brain makes secondary and tertiary prevention more difficult and early diagnosis is of utmost importance (Pulli and Chen, 2014). Non-invasive molecular imaging of key actors in neuroinflammation may be useful tools to solve this problem. Neuroinflammation can be identified even in molecular levels by using magnetic resonance imaging (MRI), positron emission tomography (PET), single photon emission computed tomography (SPECT), and optical imaging. Visualizing the adhesion molecules such as vascular adhesion molecule-1 (VCAM-1), intercellular adhesion molecule (ICAM)-1, integrin and E- and P-selectin, show endothelial cell activation, while oxidative stress, granule release and phagocytosis are related with function of the leukocytes (Pulli and Chen, 2014).

The most important consequences caused by inflammation are demyelination and neuronal death, which both may be visualized via cellular/molecular imaging technics. 11C-flumazenil is a helpful example to show the different effects of the same molecule in different conditions: In MS, at early stage, 11Cflumazenil PET is of prognostic value; neuronal loss correlates significantly with disease progression and irreversible disability. The central benzodiazepine receptor expressing neurons can be targeted with $11 \mathrm{C}$ flumazenil enhanced PET and are accepted as a good marker for neuron integrity in stroke (Sette et al., 1993, Heiss et al., 1997; Pulli and Chen, 2014). Also, in Alzheimer's disease, reduction of 11C-flumazenil binding corresponded precisely with areas of neuronal loss (Pascual et al., 2012; Pulli and Chen, 2014). Another example, "annexin-V, is a protein binding with high affinity to apoptotic cells expressing phosphatidylserine (PS) on their cell surface. With 99mTc labeled annexin-V, apoptosis successfully detected on SPECT" (Koopman et al., 1994; Pulli and Chen, 2014).

\section{NEURO- AND IMMUNO-ENGINEERING}

Neuro-engineering is one of biochemical engineering interdisciplinary areas that uses engineering techniques to understand, repair, replace or enhance neural systems. With the contribution of neuroscience, molecular biology, device development, computation, and mathematics, it has become one of the most exciting novel ventures in science and technology today. This concept brings insights from neuroscience and cognitive science with rapidly changing state-of-the-art technologies to create significant innovations inspired by or directed to the brain. Technologies for imaging, sensing, or affecting real-time brain activity and behavior, computing paradigms, augmented and adaptive systems, and brain-computer interfaces are all accounted in tools used for neuro-engineering (Figure 3).

Neuro-engineering can develop devices and sophisticated computing to assist those with neural disorders. Since, nearly one billion people worldwide are affected by traumatic or neurodegenerative pathologies, it is one of the most important tools of neuro-restoration and neuro-rehabilitation approaches (Prochazka 2017). While revealing how computations are done by natural neural systems, neuroengineering inspire new algorithms, mathematics, technologies, and robotics between engineering and living systems. Diagnostic devices, such as functional MRI examining the inner workings of the nervous system, high performance sensory devices helping to restore the missing neural function, smart prostheses competing to replace missing limbs and modulatory devices to correct nervous system dysfunction are all examples for the emerging field of "therapeutic neuro-engineering". There are also ultra high resolution and real time investigation devices observing, sensing, and monitoring. Micro-electrode array dishes or 2photon fluorescence microscopes showing the living network in action to explore the "terra incognita" of neuroscience are some of the novel inventions. 
Immuno-engineering applies engineering principles to the discovery and design of the immune system. Therapies, including engineering-based approaches, should harness the immune system and benefit from engineering optimization (Kim et al., 2019). Combining the know-how from both fields could lead to boosting of potency of vaccines, regulating tolerance and biocompatibility of medical implants, and enhancing tissue regeneration. Immuno-engineering may also help to improve the functionality and increase the lifetime of neurological devices by reducing the rejection caused by inflammation. Since that astrocytes and microglia have been identified as essential in many neuro-regenerative processes, they are assumed to be the foregoing keys in future design of drug delivery systems, implants, and scaffolds (Tsui et al., 2018), as briefly mentioned below:

\section{Nanoparticles: Nano-neuroscience}

"A nanoparticle is a microscopic particle with at least one dimension less than $100 \mathrm{~nm}$. Nanoparticle research is currently an area of intense scientific research, due to a wide variety of potential applications in biomedical, optical, and electronic fields" (Shahid et al., 2009). Design of a nanoparticle should consider the target cell, mechanism of uptake and potential uptake by phagocytic cells in the brain such as microglia (Tsui et al., 2018). An ideal nanoparticle should compose of an inert or functional vehicle. It should also traverse the BBB and selectively interact with the target. Since microglia are sensitive to nanoparticles, they also need to be able to avoid phagocytosis and clearance by microglia (Xue et al., 2012). Nanoparticles have been extensively used in neuroscience to investigate their potential applications for the diagnosis, treatment and monitoring of several neurological diseases (Berger 2019).

Nano-neuroscience research aims to "regenerate and protect the CNS by developing nanoengineered substrates, for example, to help guide axon growth after damage or degeneration. Other therapeutic strategies for CNS disorders require getting a device or drug to a specific site in the CNS" (Vidu et al., 2015). Nanomaterials may be used as vessels to deliver drugs. Nanoparticles are promising tools "to provide chips that will interface with the brain and allowing to detect and correcting online any potential miss-function of the brain's microcircuits bridging the perception with the executive control of behavior" (Vidu et al., 2015).

\section{Hydrogels}

Hydrogels are three-dimensional networks which are formed from hydrophilic polymers. They are comprised of up to $90 \%$ of water. Hydrogels could provide mechanical support and are important tools for neuroregenertion. "Hyaluronic acid, collagen type I, alginate, chitosan, methylcellulose, Matrigel, fibrin, gellan gum, self-assembling peptides and proteins, poly (ethylene glycol), methacrylates, and methacrylamides are all hydrogels which may be used in brain injury therapy or give promising results in brain injury research studies" (Kornev et al., 2018).

Hydrogels may help the axonal regeneration after traumatic brain injury. When the newly formed cavity is filled with hydrogels, they may promote axonal regeneration while providing a suitable substrate for axonal growth (Carballo and Velasco, 2015). Koss et al. introduced "basal lamina mixture into the hydrogel formula thereby demonstrating the feasibility of augmenting the resulting scaffold with bioactive substrates, and improving cell integration into the scaffold" (Koss et al, 2017). Culturing microglia in hydrogels is also feasible because of the advantageous in offering systematic and high-throughput modelling of the CNS in vitro and they can be used to model various injuries (Koss et al., 2016). In summary, hydrogels promote axonal regeneration after injury and reduce glial scarring or attenuate neuroinflammation (Koss et al., 2016), but they also may help drug release, bridging between the lesioned tissue and others and presenting models for research (George et al., 2019).

\section{Microelectrode Implants}


A neural implant, or an electrode, is a device placed inside the body and interacts with neurons via electrical impulses in particular patterns. The study on neural implants started early in 1970's (Calvello 2013). People may regain their abilities that have been lost via injuries or degenerative disorders with the help of neural devices. An electrical circuitry which is implanted as a neural device may either stimulate the nervous system or record the electrical activity of the nerve cell. "This device enhances senses, physical movement, and memory. To restore cognitive function, a neural implant must gather data from one area of the brain, process this information correctly, and then deliver the resulting signal to another brain region, bypassing any damaged tissue. In the past few decades, humans have developed advanced technologies that produced major improvements in the quality of life, their survivability, or their performance in a job. However, scientists predict that within the next twenty years neural interfaces will be designed to not only increase the dynamic range of senses, but will also enhance memory and enable cyberthink, which is invisible communication with others" (Ach and Lüttenberg, 2008).

Treating brain disorders using implants may use tools including electrode arrays, neuro-modulation chips and control module microprocessors (Figure 4). "A common and ongoing limitation of neural prosthetics is their inconsistent recording quality and long-term stability due to neuro-inflammation which is altering electrical and chemical activity around implanted probes" (Tsui et al., 2019). Following an implantable device, microglia become activated and call astrocytes to recruit at the site of implant. This reaction is called astroogliosis resulting with a scar formation (Tsui et al. 2019). Activation of microglia and astrocytes stimulate the production of prooinflammatory cytokines such as IL-1, IL-6 and TNF, which inhibit the regeneration process and support excessive cell death. The encapsulated layer around the implant is also produced by the activated microglia and astrocytes and limits regeneration (Tsui et al., 2019). Implantation of neural probes leads to overproduction of reactive oxygen species which stimulate the foreign body response, and facilitate neuronal death, corrosion and delamination of the microelectrode surface (Ereifej et al., 2018).

"An in vitro model of glial scarring around neuroelectrodes chronically implanted in CNS" (Polikov et al., 2006), was developed either by using mechanical scrape or microwire as foreign body placement in a primary cell-based system. Polikov et al. evaluated the roles of microglia and astrocytes in a cell culture implant model $6 \mathrm{~h}$ and 10 days. Scrape wound was used for acute and microwire insertion was used for chronic implantation. Although microglia invaded the scraped wound at early timepoints, activated astrocytes came to the lesion area first after 7 days. A glial scar developed at day 10 of the microwire insertion with a microglial core in the middle and surrounding astrocytes around. During the implantation process neurons were electrically active but did not repopulate around the lesion (Polikov et al., 2006). Primary glial cell responses observed in vivo were correlated well with cell culture. The surface material coating the electrode and implant duration does also matter by distribution and quantification of especially microglia on the surface of retrieved microelectrodes (Leung et al., 2008). According to the results of these studies, understanding the role of glial cells in implantable device rejection would help gain several improvements in material design (Polikov et al., 2006; Leung et al., 2008).

The major failure modes of microelectrode arrays are based on several problems. First, the place of replacement is important, the top of the electrode should become encased in a thin layer of the arachnoid to get stabilized (Prochazka 2017). Second, biological failure such as bleeding, hardware infection, meningitis, gliosis, meningeal encapsulation and extrusion are important to disrupt the tolerance of the immune system to the implanted device. Material and mechanical failures also regulate the glial cell coverage and may be an inducer of undesirable inflammatory activity. Mechanically adaptive electrodes may have regulatory effects on implant induced inflammation. Material stiffness, micromotion and tethering are all factors which must be considered in electrode design (Prochazka 2017). Devices which are initially rigid but become compliant after implantation show reduced neuroinflammatory response in long term implantation in addition to early reduced immune response to mechanically compliant intracortical implants (Nguyen et al., 
2014). Large diameter and tethered implants strongly influence tissue reactions in the CNS (Thelin et al., 2011).

There are several technical modifications of classical microelectrodes in current research and treatment. Cell grafting onto electrode implants can secrete neuroprotective factors into the surrounding area and potentially inhibit harmful cytotoxic and biochemical mechanisms for implantation (Gaillard et al., 2007). Compared to single electrodes, the use of microelectrode arrays can also produce relevant host response to the insertion (Nolta et al., 2015); but all these modifications need further in vitro and especially, in vivo investigation before claiming to be useful in clinics.

\section{Other Materials and Laboratory Technics in Neuroimmunoengineering}

Bioengineering research may be basically analyzed in four groups which all should be harnessed and coordinated to take part in the challenge. Drivers are important for early detection and treatment of the disease. They also may include artificial sensory organs including eyes, nose, etc. Measurements and analyses are sensing the states of individual cells. Devices such as $\mu$-energy sources and nano-delivery devices are important in bio-devices, bio-system monitoring and management. With the contribution of technologies such as wireless network and two-way interfaces with bio-tissues, intelligent microbioelectronics systems are getting stronger and resistant (Walker et al., 2009).

Drug delivery systems for neural prosthetic-induced inflammation involve the use of conducting polymers and they are preferred in many degenerative disorders as regulatory tools of the immune system (Green and Abidian, 2015). Disc-shaped polymer patches and cellular backpacks may also be used for drug delivery. Gold is another effective substrate for drug delivery coatings and also biosensor construction and nanoelectrical device coatings (Tsui et al., 2019). All these biomaterials and applications have notable advantages, but also challenges that will determine the future directions in clinics.

In the last decades bioengineering technics have opened a brilliant area for researchers in many areas including bio-medicine. In vitro and in vivo research tools have detailed many cellular and molecular pathways which give promising translational ideas for the clinics. Genomic, proteomic and cellular technics and imaging tools in every step of research are successful offsprings of neuroimmunoengineering. In addition, miniaturized lab-on-a-chip devices which integrate and automate many laboratory technics on a single chip provide cost efficiency, low volume reagents, high parallelization, ergonomy, high diagnostic speed, high sensitivity, and high expandability for researchers (Y1lmaz and Y1lmaz, 2017).

\section{CONCLUSION}

The application of engineering, especially electronics technology to biology and medicine is not a new concept. The entire medical imaging industry, cardiac pacemakers and cochlear implants had been successful examples of bioengineering for decades. In neuroscience, understanding the molecular basis of nerve and muscle function with the help of high-impedance amplifiers was another challenge in the late 1940's. These studies combining the quantitative biology and practical clinical neuroscience led directly to many Nobel Prizes in history. As a next step, stakeholders from government, academic and industry should build intelligent collaborations and interdisciplinary working groups to overcome the new challenges of the century. Progress in these sectors requires innovation in areas including measurement and characterization, fabrication, and power sources. In addition, development of nanoscale bioelectronics will be important in genomics and proteomics studies and open a new era for molecular-based personalized medicine.

Neurostimulation procedures, devices and imaging technics in global market is growing with a sharp acceleration in our century. "Government, industry, and academic leaders from different sectors and disciplines must be willing to commit to joint efforts where interdisciplinary contributions are necessary for success" (Walker et al., 2009). Moreover, this merge of systems biology, neuroscience, medicine, 
bioengineering, and neuro-rehabilitation may lead the way to a need for contribution of other interdisciplinary areas called neuro-law and neuro-ethics.

Collectively, innovative material science is anticipated for non-corrosive, durable, tolerable/unrejectable products, with higher specifity, higher sensitivity, high resolution and high-speed features. Optimized semiconductors and chips, miniaturization to the highest possible measures, and also highly compliable computer software for precise data processing and storage are all strongly needed. Finally, integrating modern engineering technics with systems biology, material science and medicine will definitely result in "equally profound quantum leaps" (Walker et al., 2009).

Brain is wider than the sky and deeper than the sea (E. Dickinson), but those who have been privileged to get to know the brain and the nervous system should also have the mission to act or interact (A. Einstein).

\section{REFERENCES}

1. Ach, J.S., Lüttenberg, B. Ethical Aspects of ICT Implants in the Human Body. Opinion of the European Group on Ethics in Science and New Technologies to the European Commision. (2008). In: Nanobiotechnology, Nanomedicine and Human Enhancement. Eds: Ach, J.S., Lüttenberg, B. LIT Verlag Münster. 157-186.

2. Bean, B.P. (2007). The action potential in mammalian central neurons. Nature Reviews Neuroscience. 8, 451-465.

3. Berger, M. (2017). Nanotechnology for neuroscience.

https://www.nanowerk.com/spotlight/spotid=4834 8.php

4. Brown, G.C., Vilalta, A. (2015). How microglia kills neurons. Brain Research. 1628, 288-297.

5. Calvello T. Human Enhancement: Brain Chips (Neural Implants).

(2013) https://humanenhancementusingbrainchips. weebly. com/neural-implants.html

6. Carballo-Molina, O.A., Velasco, I. (2015). Hydrogels as scaffolds and delivery systems to enhance axonal regeneration after injuries. Frontiers in Cellular Neuroscience. 9, 13.

7. Carrillo-Vico, A., Lardone, P.J., AlvarezSanchez, N., Rodriguez-Rodriguez, A., Guerrero, J.M. (2013). Melatonin: buffering the immune system. International Journal of Molecular Sciences.14 (4), 8638-8683. 8. Lee,K. Y., \&
Mooney, D. J. (2012). Alginate: propertis and biomedical applications. Progress in polyme science, 37(1), 106-126. https://oi.org/10.1016/j.progpolymsci.2011.06.003

8. David, S., Kroner, A., Greenhalgh, A.D., Zarruk, J.G., Lopez-Vales, R.J. (2018). Myeloid cell responses after spinal cord injury. Neuroimmunology. 321, 97-108.

9. Diamond, M.C., Scheibel, A.B., Murphy, G.M. Jr., Harvey, T. (2017). On the Brain of a Scientist: Albert Einstein. Experimental Neurology. 88, 198204.

10. Elsayed, M., Magistretti, P.J. (2015). A New Outlook on Mental Illnesses: Glial Involvement Beyond the Glue. Frontiers in Cellular Neuroscience. 9, 468.

11. Ereifej, E.S., Rial, G.M., Hermann, J.K., Smith, C.S., Meade, S.M., Rayyan, J.M., Chen. (2018). Implantation of Neural Probes in the Brain Elicits Oxidative Stress. Frontiers in Bioengineering and Biotechnology. 6:9.

12. Gaillard A., Prestoz, L., Dumartin, B., Cantereau, M., Morel, F., Roger, M., Jaber, M. (2017). Reestablishment of damaged adult motor pathways by grafted embiyonic cortical neurons. Nature Neuroscience, 10 (10), 1294-1299. 
13. Gaudet, A.D., Fonken, L.K. (2018). Glial Cells Shape Pathology and Repair After Spinal Cord Injury. Neurotherapeutics. 15 (3), 554-577.

14. George, J., Hsu, C., Nguyen, L.T.B., Ye, H., Cui, Z. (2019). Neural tissue engineering with structured hydrogels in CNS models and therapies. Biotechnology Advances. 03, 009.

15. Green, R., Abidian, M.R. (2015). Conducting polymers for neural prosthetic and neural interface applications. Advanced Materials. 27 (46), 76207637.

16. Heiss, W.D., Graf, R., Fujita, T., Ohta, K., Bauer, B., Löttgen, J., Wienhard, K. (1997). Early detection of irreversibly damaged ischemic tissue by flumazenil positron emission tomography in cats. Stroke. 28, 2045-2051.

17. Hu, X, Leak, R.K., Shi, Y., Suenaga, J., Gao, Y., Zheng, P., Chen, J. (2015). Microglial and macrophage polarization - new prospects for brain repair. Nature Reviews Neurology. 11, 56.

18. Jessen, K.R., Mirsky, R., Lloyd, A.C. (2015). Schwann cells: development and role in nerve repair. Cold Spring Harbor Perspectives in Biology. 7.

19. Kim, J.K., Shin, Y.J., Ha, L.J., Kim, D.H., Kim, D.H. (2019). Unraveling the Mechanobiology of the Immune System. Advanced Healthcare Materials. 8 (4), e1801332.

20. Kipnis, J., Filiano, A.J. (2017). The central nervous system: privileged by immune connections. Nature Reviews Immunology. 18, 83-84.

21. Koopman, G., Reutelingsperger, C.P., Kuijten, G.A., Keehnen, R.M., Pals, S.T., van Oers, M.H.J. (1994). Annexin V for flow cytometric detection of phosphatidylserine expression on B cells undergoing apoptosis. Blood. 84, 1415-1420.

22. Kornev, V.A., Grebenik, E.A., Solovieva, A.B., Dmitriev, R.I., Timashev, T.S. (2018). Hydrogelassisted neuroregeneration approaches towards brain injury therapy: A state-of-the-art review.
Computational and Structural Biotechnology Journal. 16, 488-502.

23. Koss, K.M., Unsworth, L.D. (2016). Neural tissue engineering: bioresponsive nanoscaffolds using engineered self-assembling peptides. Acta Biomaterialia. 44, 2-15.

24. Koss, K.M., Churchward, M.A., Jeffery, A.F., Mushahwar, V.K., Elias, A.L., Todd, K.G. (2017). Improved 3D hydrogel cultures of primary glial cells for in vitro modelling of neuroinflammation. Journal of Visualized Experiments. 1-11.

25. Leung, B.K., Biran, R., Underwood, C.J., Tresco, P.A. (2008). Characterization of microglial attachment and cytokine release on biomaterials of differing surface chemistry. Biomaterials. 29, 32893297.

26. Li, T., Wang, P., Wang, S.C., Wang, Y.F. (2017). Approaches mediating Oxytocin Regulation of the Immune System. Frontiers in Immunology. 7, 693.

27. Louveau, A., Harris, T.H., Kipnis, J. (2015). Revisiting the mechanisms of CNS immune privilege. Trends in Immunology. 36 (10), 569-577.

28. Neher, J.J., Neniskyte, U., Brown, G.C. (2012). Primary phagocytosis of neurons by inflamed microglia: potential roles in neurodegeneration. Frontiers in Pharmacology. 3.

29. Nguyen, J.K., Park, D.J., Skousen, J.L., HessDunning, A.E., Tyler, D.J., Rowan, S.J., Weder, C., Cpadona, J.R. (2014). Mechanically-compliant intrasortical implants reduce the neuroinflammatory response. Journal of Neural Engineering. 11 (5).

30. Nolta, N.F., Christensen, M.B., Crane, P.D., Skousen, J.L., Tresco, P.A. (2015). BBB leakage, astrogliosis, and tissue loss correlate with silicon microelectrode array recording performance. Biomaterials. 53, 753-762.

31. Pascual, B., Prieto, E., Arbizu, J., MartiCliment, J.M., Peñuelas, I., Quincoces, G., Zarauza, 
R., Pappata, S., Masdeu, J.C. (2012). Decreased carbon-11-flumazenil binding in early Alzheimer's disease. Brain. 135, 2817-2825.

32. Polikov, V.S., Block, M.L., Fellous, J.M., Hong, J.S., Reichert, W.M. (2006). In vitro model of glial scarring around neuroelectrodes chronically implanted in the CNS. Biomaterials. 27, 5368-5376.

33. Prinz, M., Priller, J. (2014). Microglia and brain macrophages in the molecular age: from origin to neuropsychiatric disease. Nature Reviews Neuroscience. 15 (5), 300-312.

34. Prochazka A. (2017). Biology of Neuroengineering Interfaces-Neurophysiology and neural engineering: a review. Journal of Neurophysiology. 118 (2), 1292-1309.

35. Pulli, B., Chen, J.W. (2014). Imaging Neuroinflammation-from Bench to Bedside. Journal of Clinical and Cellular Immunology. 5, 226.

36. Ramesh, G., MacLEan, A.G., Phillipp, M.T. (2013). Cytokines and chemokines at the crosroads of neuroinflammation, neurodegeneration, and neuropathic pain. Mediators Inflammation. 480739.

37. Sette, G., Baron, J.C., Young, A.R., Miyazawa, H., Tillet, I., Barre, L., Travere, J.M., Derlon, J.M., MacKenzie, E.T. (1993). In vivo mapping of brain benzodiazepine receptor changes by positron emission tomography after focal ischemia in the anesthetized baboon. Stroke. 24, 2046-2057.

38. Shahid, M., Khan, H.M., Mustafa, S., Shujaatullah, F. (2009). Nanotechnology: Implications of nanoparticles in medical science. In: Biotechnology Emerging Trends. Eds: Sayyed RZ, Patil AS. Scientific Publishers. pp. 529-550.

39. Sochocka, M., Diniz, B.S., Leszek, J. (2017). Inflammatory response in the CNS: Friend or Foe? Molecular Neurobiology. 54 (10), 8071-8089.

40. Thelin, J., Jörntell, H., Psouni, E., Garwicz, M., Schouenborg, J., Danielsen, N., Linsmeier, C.E. (2011). Implant size and fixation mode strongly influence tissue reactions in the CNS. PLoS One. 6.
41. ThyagaRajan, S., Priyanka, H.P. (2012). Bidirectional communication between the neuroendocrine system and the immune system: relevance to health and diseases. Annals of Neurosciences. 19 (1), 40-46.

42. Tsui, C., Koss, K., Churchward, M.A., Todd, K.G. (2019). Biomaterials and glia: Progress on designs to modulate neuroinflammation. Acta Biomaterialia. 83, 13-28.

43. Vassanelli, S., Mahmud, M. (2016). Trends and challenges in neuroengineering: toward "intelligent" neuroprotheses through brain-"brain inspired systems" communication. Frontiers in neuroscience. 10, 438 .

44. Vidu, R., Rahman, M., Mahmoudi, M., Enaschescu, M., Poteca, D.T., Opris, I. (2015). Nanostructures: a platform for brain repair and augmentation. Frontiers in Systems Neuroscience. 8,91 .

45. Walker, G.M., Ramsey, J.M., Cavin, R.K., Herr, D.J.C., Merzbacher, C.I., Zhirnov, V. (2009). https://www.nist.gov/system/files/documents/pml/ div683/bioelectronics_report.pdf

46. Xue, D., Zhao, M., Wang, Y.J., Wang, L., Yang, Y., Wang, S.W., Zhang, R., Zhao, Y., Liu, R.T. (2012). A multifunctional peptide rescues memory deficits in Alzheimer's disease transgenic mice by inhibiting A $\beta 42$-induced cytotoxicity and increasing microglial phagocytosis. Neurobiology of Disease. 46 (3), 701-709.

47. Yilmaz B, Yilmaz F. (2017). Lab-on-a-chip technology and its application. In: Omics Technologies and Bioengineering. Eds: Brah D, Azevedo V. Elsevier. pp. 145-153. 